\title{
Solving the incompressible surface Navier-Stokes equation by surface finite elements
}

\author{
Sebastian Reuther ${ }^{\mathrm{a}}$, Axel Voigt ${ }^{\mathrm{a}, \mathrm{b}, \mathrm{c}}$ \\ ${ }^{a}$ Department of Mathematics, TU Dresden, Dresden, Germany \\ ${ }^{b}$ Dresden Center for Computational Materials Science (DCMS), Dresden, Germany \\ ${ }^{c}$ Center for Systems Biology Dresden (CSBD), Dresden, Germany
}

\begin{abstract}
We consider a numerical approach for the incompressible surface NavierStokes equation on surfaces with arbitrary genus $g(\mathcal{S})$. The approach is based on a reformulation of the equation in Cartesian coordinates of the embedding $\mathbb{R}^{3}$, penalization of the normal component, a Chorin projection method and discretization in space by surface finite elements for each component. The approach thus requires only standard ingredients which most finite element implementations can offer. We compare computational results with discrete exterior calculus (DEC) simulations on a torus and demonstrate the interplay of the flow field with the topology by showing realizations of the PoincaréHopf theorem on $n$-tori.
\end{abstract}

Keywords: interfacial flow, surface viscosity, surface finite elements, vortex dynamics, Chorin projection

\section{Introduction}

We consider a compact smooth Riemannian surface $\mathcal{S}$ without boundary and an incompressible surface Navier-Stokes equation

$$
\begin{aligned}
\partial_{t} \mathbf{v}+\nabla_{\mathbf{v}} \mathbf{v} & =-\operatorname{grad}_{\mathcal{S}} p+\frac{1}{\operatorname{Re}}\left(-\boldsymbol{\Delta}^{\mathrm{dR}} \mathbf{v}+2 \kappa \mathbf{v}\right) \\
\operatorname{div}_{\mathcal{S}} \mathbf{v} & =0
\end{aligned}
$$

in $\mathcal{S} \times(0, \infty)$ with initial condition $\mathbf{v}(\mathbf{x}, t=0)=\mathbf{v}_{0}(\mathbf{x}) \in \mathrm{T}_{\mathbf{x}} \mathcal{S}$. Thereby $\mathbf{v}(t) \in \mathrm{T} \mathcal{S}$ denotes the tangential surface velocity, $p(\mathbf{x}, t) \in \mathbb{R}$ the surface pressure, Re the surface Reynolds number, $\kappa$ the Gaussian curvature, $\mathrm{T}_{\mathbf{x}} \mathcal{S}$ 
the tangent space on $\mathbf{x} \in \mathcal{S}, \mathrm{T} \mathcal{S}=\cup_{\mathbf{x} \in \mathcal{S}} \top_{\mathbf{x}} \mathcal{S}$ the tangent bundle and $\nabla_{\mathbf{v}}$, $\operatorname{div}_{\mathcal{S}}$ as well as $\boldsymbol{\Delta}^{\mathrm{dR}}$ the covariant directional derivative, surface divergence and surface Laplace-deRham operator, respectively. The surface Navier-Stokes equation results from conservation of mass and (tangential) linear momentum. Alternatively, eqs. (1) and (2) can also be derived from the Rayleigh dissipation potential Dörries and Foltin (1996) or as a thin-film limit of the three-dimensional incompressible Navier-Stokes equation Miura (2017).

The incompressible surface Navier-Stokes equation is related to the Boussinesq-Scriven constitutive law for the surface viscosity in two-phase flow problems Scriven (1960); Secomb and Skalak (1982); Bothe and Prüss (2010) and to fluidic biomembranes $\mathrm{Hu}$ et al. (2007); Arroyo and DeSimone (2009); Barrett et al. (2015); Reuther and Voigt (2016). Further applications can be found in computer graphics, e.g. Elcott et al. (2007); Mullen et al. (2009); Vaxman et al. (2016), and geophysics, e.g. Padberg-Gehle et al. (2017); Sasaki et al. (2015). The equation is also studied as a mathematical problem of its own interest, see e.g. Ebin and Marsden (1970); Mitrea and Taylor (2001).

While a huge literature exists for the numerical treatment of the twodimensional incompressible Navier-Stokes equation in flat space, results for its surface counterpart eqs. (1) and (2) are rare. In Nitschke et al. (2012); Reuther and Voigt (2015) a surface vorticity-stream function formulation is introduced. However, this approach cannot deal with harmonic vector fields and is therefore only applicable on surfaces with genus $g(\mathcal{S})=0$. The only direct numerical approach for eqs. (1) and (2), which is also desirable for surfaces with genus $g(\mathcal{S}) \neq 0$, was proposed in Nitschke et al. (2017) and uses discrete exterior calculus (DEC).

The purpose of this paper is to introduce a surface finite element discretization with only standard ingredients. This is achieved by extending the variational space from vectors in $T \mathcal{S}$ to vectors in $\mathbb{R}^{3}$ and penalizing the normal component. This allows to split the vector-valued problem into a set of coupled scalar-valued problems for each component for which standard surface finite elements, see the review Dziuk and Elliott (2013), can be used. Similar approaches have already been independently used for other vectorvalued problems, see Hansbo et al. (2016) for a surface vector Laplacian, Nestler et al. (2017) for a surface Frank-Oseen problem and Jankuhn et al. (2017) for a surface Stokes problem.

The paper is organized as follows. In Section 2 we introduce the necessary notation, reformulate the problem in Cartesian coordinates of the 
embedding $\mathbb{R}^{3}$ and introduce the penalization of the normal component. We further modify the equation by rotating the velocity field, which reduces the complexity of the equation. In Section 3 we describe the numerical approach. For the resulting equations we propose a Chorin projection approach and a discretization in space by standard piecewise linear Lagrange surface finite elements. We demonstrate the reduction of computational time due to the introduced rotation and validate our approach against a DEC solution on a torus with harmonic vector fields, see Nitschke et al. (2017). In Section 4 results are shown and analyzed on $n$-tori and conclusions are drawn in Section 5 .

\section{Model formulation}

We follow the same notation as introduced in Nestler et al. (2017) and parametrize the surface $\mathcal{S} \subset \mathbb{R}^{3}$ by the local coordinates $\theta, \varphi$, i. e.,

$$
\mathbf{x}: \mathbb{R}^{2} \supset U \rightarrow \mathbb{R}^{3} ;(\theta, \varphi) \mapsto \mathbf{x}(\theta, \varphi) .
$$

Thus, the embedded $\mathbb{R}^{3}$ representation of the surface is given by $\mathcal{S}=\mathbf{x}(U)$. The unit outer normal of $\mathcal{S}$ at point $\mathbf{x}$ is denoted by $\boldsymbol{\nu}(\mathbf{x})$. We denote by $\left\{\partial_{\theta} \mathbf{x}, \partial_{\varphi} \mathbf{x}\right\}$ the canonical basis to describe the (tangential) velocity $\mathbf{v}(\mathbf{x}) \in$ $\mathrm{T}_{\mathbf{x}} \mathcal{S}$, i. e., $\mathbf{v}=v^{\theta} \partial_{\theta} \mathbf{x}+v^{\varphi} \partial_{\varphi} \mathbf{x}$ at a point $\mathbf{x} \in \mathcal{S}$. In a (tubular) neighborhood $\Omega_{\delta}$ of $\mathcal{S}$, defined by $\Omega_{\delta}:=\left\{\widetilde{\mathbf{x}} \in \mathbb{R}^{3}: d_{\mathcal{S}}(\widetilde{\mathbf{x}})<\frac{1}{2} \delta\right\}$, with a signed-distance function $d_{\mathcal{S}}(\widetilde{\mathbf{x}})$ a coordinate projection $\mathbf{x} \in \mathcal{S}$ of $\widetilde{\mathbf{x}} \in \mathbb{R}^{3}$ is introduced, such that $\widetilde{\mathbf{x}}=\mathbf{x}+d_{\mathcal{S}}(\widetilde{\mathbf{x}}) \boldsymbol{\nu}(\mathbf{x})$. For $\delta$ sufficiently small (depending on the local curvature of the surface) this projection is injective, see Dziuk and Elliott (2013). For a given $\widetilde{\mathbf{x}} \in \Omega_{\delta}$ the coordinate projection of $\widetilde{\mathbf{x}}$ will also be called gluing map, denoted by $\pi: \Omega_{\delta} \rightarrow \mathcal{S}, \widetilde{\mathbf{x}} \mapsto \mathbf{x}$. The pressure $p: \mathcal{S} \rightarrow \mathbb{R}$ and the velocity $\mathbf{v}: \mathcal{S} \rightarrow \mathrm{T} \mathcal{S}$ can be smoothly extended in the neighborhood $\Omega_{\delta}$ of $\mathcal{S}$ by utilizing the coordinate projection, i. e., extended fields $\tilde{p}: \Omega_{\delta} \rightarrow \mathbb{R}$ and $\tilde{\mathbf{v}}: \Omega_{\delta} \rightarrow \mathbb{R}^{3}$ are defined by

$$
\tilde{p}(\widetilde{\mathbf{x}}):=p(\mathbf{x}) \quad \text { and } \quad \tilde{\mathbf{v}}(\widetilde{\mathbf{x}}):=\mathbf{v}(\mathbf{x}),
$$

respectively, for $\widetilde{\mathbf{x}} \in \Omega_{\delta}$ and $\mathbf{x}$ the corresponding coordinate projection. To embed the $\mathbb{R}^{3}$ vector space structure to the tangential bundle of the surface we use the pointwise defined normal projection

$$
\begin{aligned}
\pi_{\mathcal{S}}(\mathbf{x}): \mathrm{T}_{\mathbf{x}} \mathbb{R}^{3} \cong \mathbb{R}^{3} & \rightarrow \mathrm{T}_{\mathbf{x}} \mathcal{S} ; \\
\widehat{\mathbf{v}}(\mathbf{x}) & \mapsto \widehat{\mathbf{v}}(\mathbf{x})-\boldsymbol{\nu}(\mathbf{x})(\boldsymbol{\nu}(\mathbf{x}) \cdot \widehat{\mathbf{v}}(\mathbf{x}))=\mathbf{v}(\mathbf{x})
\end{aligned}
$$


for all $\mathbf{x} \in \mathcal{S}$, which maps the $\mathbb{R}^{3}$ velocity $\widehat{\mathbf{v}}=v_{x} \mathbf{e}^{x}+v_{y} \mathbf{e}^{y}+v_{z} \mathbf{e}^{z} \in$ $\mathbb{R}^{3}$, not necessarily tangential to the surface, to the tangential velocity $\mathbf{v} \in$ $\mathrm{T}_{\mathrm{x}} \mathcal{S}$. We drop the argument $\mathrm{x}$ when applied to velocity fields living on $\mathcal{S}$. With these notations we have the following correspondence of the different representations of first order differential operators on surfaces:

\begin{tabular}{lllll}
$\mathrm{TS}$ & $\operatorname{grad}_{\mathcal{S}} p$ & $\operatorname{rot}_{\mathcal{S}} p$ & $\operatorname{div}_{\mathcal{S}} \mathbf{v}$ & $\operatorname{rot}_{\mathcal{S}} \mathbf{v}$ \\
\hline $\mathbb{R}^{3}$ & $\pi_{\mathcal{S}} \nabla p$ & $\boldsymbol{\nu} \times \nabla p$ & $\nabla \cdot \widehat{\mathbf{v}}-\boldsymbol{\nu} \cdot(\nabla \widehat{\mathbf{v}} \cdot \boldsymbol{\nu})-\mathcal{H}(\widehat{\mathbf{v}} \cdot \boldsymbol{\nu})$ & $(\nabla \times \widehat{\mathbf{v}}) \cdot \boldsymbol{\nu}$
\end{tabular}

Thereby $\mathcal{H}$ denotes the mean curvature. We further $\operatorname{define} \operatorname{div}_{\mathcal{S}} \widehat{\mathbf{v}}=\nabla \cdot \widehat{\mathbf{v}}-$ $\boldsymbol{\nu} \cdot(\nabla \widehat{\mathbf{v}} \cdot \boldsymbol{\nu}), \operatorname{rot}_{\mathcal{S}} \widehat{\mathbf{v}}=-\operatorname{div}_{\mathcal{S}}(\boldsymbol{\nu} \times \widehat{\mathbf{v}})$ and . Using the definition of Abraham et al. (1988) the surface Laplace-deRham operator is defined as $\boldsymbol{\Delta}^{\mathrm{dR}} \mathbf{v}=$ $-\left(\boldsymbol{\Delta}^{\mathrm{RR}}+\boldsymbol{\Delta}^{\mathrm{GD}}\right) \mathbf{v}$ with $\boldsymbol{\Delta}^{\mathrm{RR}} \mathbf{v}=\operatorname{rot}_{\mathcal{S}} \operatorname{rot}_{\mathcal{S}} \mathbf{v}$ and $\boldsymbol{\Delta}^{\mathrm{GD}} \mathbf{v}=\operatorname{grad}_{\mathcal{S}} \operatorname{div}_{\mathcal{S}} \mathbf{v}$. As shown in Nestler et al. (2017) it holds

$$
\Delta^{\mathrm{dR}} \mathbf{v} \approx \widehat{\boldsymbol{\Delta}}^{\mathrm{dR}} \widehat{\mathbf{v}} \quad \text { with } \quad \widehat{\boldsymbol{\Delta}}^{\mathrm{dR}} \widehat{\mathbf{v}}=-\left(\operatorname{rot}_{\mathcal{S}} \operatorname{rot}_{\mathcal{S}} \widehat{\mathbf{v}}+\operatorname{grad}_{\mathcal{S}} \operatorname{div}_{\mathcal{S}} \widehat{\mathbf{v}}\right)
$$

if the normal component $(\widehat{\mathbf{v}} \cdot \boldsymbol{\nu})$ is penalized by the additional term $\alpha(\boldsymbol{\nu} \cdot \widehat{\mathbf{v}}) \boldsymbol{\nu}$. First order convergence in the penalty parameter $\alpha$ was numerically shown for this approximation in Nestler et al. (2017). Due to the incompressibility we here have $\boldsymbol{\Delta}^{\mathrm{dR}} \mathbf{v} \approx-\operatorname{rot}_{\mathcal{S}} \operatorname{rot}_{\mathcal{S}} \widehat{\mathbf{v}}$. With $\nabla_{\mathbf{v}} \mathbf{v}=\frac{1}{2} \operatorname{grad}_{\mathcal{S}}(\widehat{\mathbf{v}} \cdot \widehat{\mathbf{v}})+\operatorname{rot}_{\mathcal{S}} \widehat{\mathbf{v}} \boldsymbol{\nu} \times \widehat{\mathbf{v}}$ we thus obtain the approximation of the surface incompressible Navier-Stokes equation in Cartesian coordinates which ensures the velocity to be tangential only weakly through the added penalty term

$$
\begin{aligned}
\partial_{t} \widehat{\mathbf{v}}+\operatorname{rot}_{\mathcal{S}} \widehat{\mathbf{v}} \boldsymbol{\nu} \times \widehat{\mathbf{v}} & =-\operatorname{grad}_{\mathcal{S}} \tilde{p}+\frac{1}{\operatorname{Re}}\left(-\operatorname{rot}_{\mathcal{S}} \operatorname{rot}_{\mathcal{S}} \widehat{\mathbf{v}}+2 \kappa \widehat{\mathbf{v}}\right)-\alpha(\widehat{\mathbf{v}} \cdot \boldsymbol{\nu}) \boldsymbol{\nu} \\
\operatorname{div}_{\mathcal{S}} \widehat{\mathbf{v}} & =0
\end{aligned}
$$

with $\tilde{p}=p+\frac{1}{2} \widehat{\mathbf{v}} \cdot \widehat{\mathbf{v}}$. Eqs. (4) and (5) can now be solved for each component $v_{x}, v_{y}, v_{z}$ and $\tilde{p}$ using standard approaches for scalar-valued problems on surfaces, such as the surface finite element method Dziuk and Elliott (2007b a, 2013), level set approaches Bertalmio et al. (2001); Greer et al. (2006); Stöcker and Voigt (2008); Dziuk and Elliott (2008) or diffuse interface approximations Rätz and Voigt (2006). However, the $\operatorname{rot}_{\mathcal{S}} \operatorname{rot}_{\mathcal{S}} \widehat{\mathbf{v}}$ term leads to a heavy workload in terms of implementation and assembly time, as 36 second order operators, 72 first order operators and 36 zero order operators have to be considered. This effort can drastically be reduced by rotating 
the velocity field in the tangent plane. Instead of $\widehat{\mathbf{v}}$ we consider $\widehat{\mathbf{w}}=\boldsymbol{\nu} \times \widehat{\mathbf{v}}$ as unknown. Applying $\boldsymbol{\nu} \times$ to eq. (4) we thus obtain

$$
\partial_{t} \widehat{\mathbf{w}}+\operatorname{div}_{\mathcal{S}} \widehat{\mathbf{w}} \boldsymbol{\nu} \times \widehat{\mathbf{w}}=-\operatorname{rot}_{\mathcal{S}} \tilde{p}+\frac{1}{\operatorname{Re}}\left(\operatorname{grad}_{\mathcal{S}} \operatorname{div}_{\mathcal{S}} \widehat{\mathbf{w}}+2 \kappa \widehat{\mathbf{w}}\right)-\alpha(\widehat{\mathbf{w}} \cdot \boldsymbol{\nu}) \boldsymbol{\nu}
$$

$$
\operatorname{rot}_{\mathcal{S}} \widehat{\mathbf{w}}=0
$$

where we have used the identities $\operatorname{rot}_{\mathcal{S}} \widehat{\mathbf{v}}=-\operatorname{div}_{\mathcal{S}} \widehat{\mathbf{w}}, \operatorname{div}_{\mathcal{S}} \widehat{\mathbf{v}}=\operatorname{rot}_{\mathcal{S}} \widehat{\mathbf{w}}, \widehat{\mathbf{v}}=$ $-\boldsymbol{\nu} \times \widehat{\mathbf{w}}$ and $\boldsymbol{\nu} \times(\boldsymbol{\nu} \times \widehat{\mathbf{v}})=-\widehat{\mathbf{v}}$. The $\operatorname{grad}_{\mathcal{S}} \operatorname{div}_{\mathcal{S}} \widehat{\mathbf{w}}$ term now contains only 9 second order terms and the remaining terms are of similar complexity as in eqs. (4) and (5).

\section{Discretization}

\subsection{Time discretization}

Let $0<t_{0}<t_{1}<\ldots$ be a sequence of discrete times with time step width $\tau_{n}:=t_{n+1}-t_{n}$ in the $n$-th iteration. The fields $\widehat{\mathbf{v}}^{n}(\mathbf{x})$ e.g.uiv $\widehat{\mathbf{v}}\left(\mathbf{x}, t_{n}\right)$, $\widehat{\mathbf{w}}^{n}(\mathbf{x})$ e.g.uiv $\widehat{\mathbf{w}}\left(\mathbf{x}, t_{n}\right)$ and $\tilde{p}^{n}(\mathbf{x})$ e.g.uiv $\tilde{p}\left(\mathbf{x}, t_{n}\right)$ correspond to the time-discrete functions at $t_{n}$. Applying a Chorin projection method Chorin (1968) to eqs. (4) and (5) with a semi-implicit Euler time scheme results in time discrete systems of equations as follows:

Problem 1. Let $\widehat{\mathbf{v}}^{0} \in C\left(\mathcal{S} ; \mathbb{R}^{3}\right)$ be a given initial velocity field with $\widehat{\mathbf{v}}^{0}=\mathbf{v}^{0}$. For $n=0,1,2, \ldots$ find

1. $\widehat{\mathbf{v}}^{*}$ such that

$$
\frac{1}{\tau_{n}}\left(\widehat{\mathbf{v}}^{*}-\widehat{\mathbf{v}}^{n}\right)=-\operatorname{rot}_{\mathcal{S}} \widehat{\mathbf{v}}^{*} \boldsymbol{\nu} \times \widehat{\mathbf{v}}^{n}+\frac{1}{R e}\left(-\operatorname{rot}_{\mathcal{S}} \operatorname{rot}_{\mathcal{S}} \widehat{\mathbf{v}}^{*}+2 \kappa \widehat{\mathbf{v}}^{*}\right)-\alpha\left(\widehat{\mathbf{v}}^{*} \cdot \boldsymbol{\nu}\right) \boldsymbol{\nu}
$$

2. $\tilde{p}^{n+1}$ such that

$$
\tau_{n} \Delta_{\mathcal{S}} \tilde{p}^{n+1}=\operatorname{div}_{\mathcal{S}} \widehat{\mathbf{v}}^{*}
$$

3. $\widehat{\mathbf{v}}^{n+1}$ such that

$$
\widehat{\mathbf{v}}^{n+1}=\widehat{\mathbf{v}}^{*}-\tau_{n} \operatorname{grad}_{\mathcal{S}} \tilde{p}^{n+1},
$$

with $\Delta_{\mathcal{S}}$ the Laplace-Beltrami operator. 
The corresponding scheme for eqs. (6) and (7) follows by defining $\widehat{\mathbf{w}}^{*}=$ $\boldsymbol{\nu} \times \widehat{\mathbf{v}}^{*}$ and applying $\boldsymbol{\nu} \times$ to the equation in the first step. We thus obtain:

Problem 2. Let $\widehat{\mathbf{v}}^{0} \in C\left(\mathcal{S} ; \mathbb{R}^{3}\right)$ be a given initial velocity field with $\widehat{\mathbf{v}}^{0}=\mathbf{v}^{0}$. Compute $\widehat{\mathbf{w}}^{0}=\boldsymbol{\nu} \times \widehat{\mathbf{v}}^{0}$. For $n=0,1,2, \ldots$ find

1. $\widehat{\mathbf{w}}^{*}$ such that

$\frac{1}{\tau_{n}}\left(\widehat{\mathbf{w}}^{*}-\widehat{\mathbf{w}}^{n}\right)=\operatorname{div}_{\mathcal{S}} \widehat{\mathbf{w}}^{*} \boldsymbol{\nu} \times \widehat{\mathbf{w}}^{n}+\frac{1}{R e}\left(\operatorname{grad}_{\mathcal{S}} \operatorname{div}_{\mathcal{S}} \widehat{\mathbf{w}}^{*}+2 \kappa \widehat{\mathbf{w}}^{*}\right)-\alpha\left(\widehat{\mathbf{w}}^{*} \cdot \boldsymbol{\nu}\right) \boldsymbol{\nu}$

2. $\tilde{p}^{n+1}$ such that

$$
\tau_{n} \Delta_{\mathcal{S}} \tilde{p}^{n+1}=\operatorname{rot}_{\mathcal{S}} \widehat{\mathbf{w}}^{*}
$$

3. $\widehat{\mathbf{w}}^{n+1}$ such that

$$
\widehat{\mathbf{w}}^{n+1}=\widehat{\mathbf{w}}^{*}-\tau_{n} \operatorname{rot}_{\mathcal{S}} \tilde{p}^{n+1}
$$

4. $\widehat{\mathbf{v}}^{n+1}=-\boldsymbol{\nu} \times \widehat{\mathbf{w}}^{n+1}$.

For simplicity we consider only a Taylor-0 linearization of the nonlinear term in both problems.

\subsection{Space discretization}

For the discretization in space we apply the surface finite element method for scalar-valued problems Dziuk and Elliott (2013) for each component. Therefore, the surface $\mathcal{S}$ is discretized by a conforming triangulation $\mathcal{S}_{h}$, given as the union of simplices, i. e., $\mathcal{S}_{h}:=\bigcup_{T \in \mathcal{T}} T$. We use globally continuous, piecewise linear Lagrange surface finite elements

$$
\mathbb{V}_{h}\left(\mathcal{S}_{h}\right)=\left\{v_{h} \in C^{0}\left(\mathcal{S}_{h}\right):\left.v_{h}\right|_{T} \in \mathbb{P}^{1}, \forall T \in \mathcal{T}\right\}
$$

as trial and test space for all components $\widehat{v}_{i}$ of $\widehat{\mathbf{v}}$ as well as $\widehat{w}_{i}$ of $\widehat{\mathbf{w}}$ and $\tilde{p}$ with $\mathcal{T}$ the set of triangular faces.

The resulting fully discrete problem for PROBLEM 1 reads: For $n=$ $0,1,2, \ldots$ find $\widehat{v}_{i}^{*}, \tilde{p}^{n+1} \in \mathbb{V}_{h}\left(\mathcal{S}_{h}\right)$ s.t. $\forall \widehat{u}_{i}, \widehat{q} \in \mathbb{V}_{h}\left(\mathcal{S}_{h}\right)$

$$
\begin{aligned}
& \frac{1}{\tau_{n}} \int_{\mathcal{S}_{h}} \widehat{v}_{i}^{*} \widehat{u}_{i} \mathrm{~d} \mathcal{S}+\int_{\mathcal{S}_{h}} \operatorname{rot}_{\mathcal{S}} \widehat{\mathbf{v}}^{*}\left(\boldsymbol{\nu} \times \widehat{\mathbf{v}}^{n}\right)_{i} \widehat{u}_{i} \mathrm{~d} \mathcal{S}+\alpha \int_{\mathcal{S}_{h}} \boldsymbol{\nu} \cdot \widehat{\mathbf{v}}^{*} \nu_{i} \widehat{u}_{i} \mathrm{~d} \mathcal{S} \\
& \quad-\frac{1}{\operatorname{Re}} \int_{\mathcal{S}_{h}} \operatorname{rot}_{\mathcal{S}} \widehat{\mathbf{v}}^{*} \operatorname{rot}_{\mathcal{S}}\left(\widehat{u}_{i} \mathbf{e}^{i}\right) \mathrm{d} \mathcal{S}-2 \int_{\mathcal{S}_{h}} \kappa \widehat{v}_{i}^{*} \widehat{u}_{i} \mathrm{~d} \mathcal{S}=\frac{1}{\tau_{n}} \int_{\mathcal{S}_{h}} \widehat{v}_{i}^{n} \widehat{u}_{i} \mathrm{~d} \mathcal{S} \\
& \tau_{n} \int_{\mathcal{S}_{h}} \operatorname{grad}_{\mathcal{S}} \tilde{p}^{n+1} \cdot \operatorname{grad}_{\mathcal{S}} \widehat{q} \mathrm{~d} \mathcal{S}+\int_{\mathcal{S}_{h}} \widehat{\mathbf{v}}^{*} \cdot \operatorname{grad}_{\mathcal{S}} \widehat{q} \mathrm{~d} \mathcal{S}=0
\end{aligned}
$$


for $i=x, y, z$, from which $\widehat{\mathbf{v}}^{n+1}$ can be computed according to step 3 in PROBLEM 1,

The resulting fully discrete problem for PROBLEM 2 reads: For $n=$ $0,1,2, \ldots$ find $\widehat{w}_{i}^{*}, \tilde{p}^{n+1} \in \mathbb{V}_{h}\left(\mathcal{S}_{h}\right)$ s.t. $\forall \widehat{u}_{i}, \widehat{q} \in \mathbb{V}_{h}\left(\mathcal{S}_{h}\right)$

$$
\begin{aligned}
& \frac{1}{\tau_{n}} \int_{\mathcal{S}_{h}} \widehat{w}_{i}^{*} \widehat{u}_{i} \mathrm{~d} \mathcal{S}-\int_{\mathcal{S}_{h}} \operatorname{div}_{\mathcal{S}} \widehat{\mathbf{w}}^{*}\left(\boldsymbol{\nu} \times \widehat{\mathbf{w}}^{n}\right)_{i} \widehat{u}_{i} \mathrm{~d} \mathcal{S}+\alpha \int_{\mathcal{S}_{h}} \boldsymbol{\nu} \cdot \widehat{\mathbf{w}}^{*} \nu_{i} \widehat{u}_{i} \mathrm{~d} \mathcal{S} \\
& \quad+\frac{1}{\operatorname{Re}} \int_{\mathcal{S}_{h}} \operatorname{div}_{\mathcal{S}} \widehat{\mathbf{w}}^{*}\left(\operatorname{grad}_{\mathcal{S}} \widehat{u}_{i}\right)_{i} \mathrm{~d} \mathcal{S}-2 \int_{\mathcal{S}_{h}} \kappa \widehat{w}_{i}^{*} \widehat{u}_{i} \mathrm{~d} \mathcal{S}=\frac{1}{\tau_{n}} \int_{\mathcal{S}_{h}} \widehat{w}_{i}^{n} \widehat{u}_{i} \mathrm{~d} \mathcal{S} \\
& \tau_{n} \int_{\mathcal{S}_{h}} \operatorname{grad}_{\mathcal{S}} \tilde{p}^{n+1} \cdot \operatorname{grad}_{\mathcal{S}} \widehat{q} \mathrm{~d} \mathcal{S}+\int_{\mathcal{S}_{h}} \boldsymbol{\nu} \times \widehat{\mathbf{w}}^{*} \cdot \operatorname{grad}_{\mathcal{S}} \widehat{q} \mathrm{~d} \mathcal{S}=0
\end{aligned}
$$

for $i=x, y, z$, from which $\widehat{\mathbf{w}}^{n+1}$ and $\widehat{\mathbf{v}}^{n+1}$ can be computed according to step 3 and 4 in PROBLEM 2,

To assemble and solve the resulting system we use the FEM-toolbox AMDiS Vey and Voigt (2007); Witkowski et al. (2015) with domain decomposition on 16 processors. As linear solver we have used a $\operatorname{BiCGStab}(l)$ method with $l=2$ and a Jacobi preconditioner with ILU(0) local solver on each partition.

\subsection{Comparison and validation}

Both approaches lead to the same results. However, the computational cost for PROBLEM 2 is drastically reduced. To quantify this reduction we compare the assembly time for the second order operators in PROBLEM 1 and Problem 2. We consider a sphere as computational domain $\mathcal{S}=\mathbb{S}^{2}$ and vary the triangulation $\mathcal{T}$. Figure 1 shows the assembly time as a function of degrees of freedom (DOFs). The time is the mean value of multiply runs of the assembly routine. The results indicate a reduction by a factor of approximately 50 .

We now compare the solution of PROBLEM 2 with an example considered in Nitschke et al. (2017) using DEC. It considers a nontrivial solution with $\operatorname{div}_{\mathcal{S}} \mathbf{v}=0$ and $\operatorname{rot}_{\mathcal{S}} \mathbf{v}=0$. Such harmonic vector fields can exist on surfaces with $g(\mathcal{S}) \neq 0$. We consider a torus which has genus $g(\mathcal{S})=1$. A torus can be described by the levelset function $T(\mathbf{x})=\left(x^{2}+y^{2}+z^{2}+R^{2}-r^{2}\right)^{2}-4 R^{2}\left(x^{2}+z^{2}\right)$ with $\mathbf{x}=(x, y, z) \in \mathbb{R}^{3}$, major radius $R$ and minor radius $r$. We here use $R=2$ and $r=0.5$. Let $\phi$ and $\theta$ denote the standard parametrization 


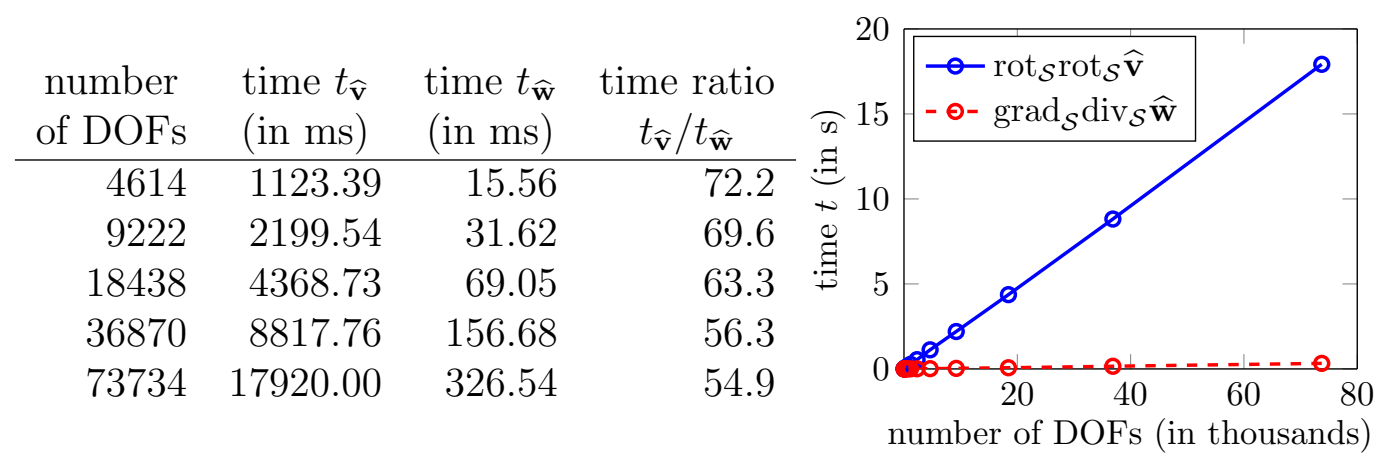

Figure 1: Assembly times $t_{\widehat{\mathbf{v}}}$ and $t_{\widehat{\mathbf{w}}}$ for the two second order operators $\operatorname{rot}_{\mathcal{S}} \operatorname{rot}_{\mathcal{S}} \widehat{\mathbf{v}}$ and $\operatorname{grad}_{\mathcal{S}} \operatorname{div}_{\mathcal{S}} \widehat{\mathbf{w}}$ as a function of the number of DOFs.

angles on the torus. Then, the two basis vectors can be written as $\partial_{\phi} \mathbf{x}$ as well as $\partial_{\theta} \mathbf{x}$ and read in Cartesian coordinates $\partial_{\phi} \mathbf{x}=(-z, 0, x)$ as well as $\partial_{\theta} \mathbf{x}=\left(-\frac{x y}{\sqrt{x^{2}+z^{2}}}, \sqrt{x^{2}+z^{2}}-2,-\frac{y z}{\sqrt{x^{2}+z^{2}}}\right)$. There are two (linear independent) harmonic vector fields on the torus,

$$
\mathbf{v}_{\phi}^{\text {harm }}=\frac{1}{4\left(x^{2}+z^{2}\right)} \partial_{\phi} \mathbf{x} \quad \text { and } \quad \mathbf{v}_{\theta}^{\text {harm }}=\frac{1}{2 \sqrt{x^{2}+z^{2}}} \partial_{\theta} \mathbf{x} .
$$

The example considers the mean of the two harmonic vector fields as initial condition $\mathbf{v}_{0}(\mathbf{x})=\frac{1}{2}\left(\mathbf{v}_{\phi}^{\text {harm }}+\mathbf{v}_{\theta}^{\text {harm }}\right)$ and shows the evolution towards a Killing vector field which is proportional to the basis vector $\partial_{\phi} \mathbf{x}$. The surface Reynolds number is $\operatorname{Re}=10$. Figure 2 shows the results obtained with the fully discrete scheme of PROBLEM 2 with time step width $\tau_{n}=0.1$ and penalization parameter $\alpha=3000$ on the same mesh as considered in Nitschke et al. (2017). For the Gaussian curvature $\kappa$ we use the analytic formula.

In figure 3 (left) we compare $\widehat{\mathbf{v}}$ with $\widehat{\mathbf{v}}^{\mathrm{DEC}}$ for various $\alpha$. Thereby $\widehat{\mathbf{v}}^{\mathrm{DEC}}$ is the solution $\mathbf{v}$ of eqs. (1) and (2) with zero normal component from Nitschke et al. (2017). Again first order convergence in $\alpha$ can be obtained. In figure 3 (right) we consider the rescaled velocity field $\widehat{\mathbf{v}}^{*}=\widehat{\mathbf{v}} /\|\widehat{\mathbf{v}}\|_{L^{2}}$ in order to show that the penalization of the normal component $\widehat{\mathbf{v}}^{*} \cdot \boldsymbol{\nu}$ is numerically satisfied. For $\alpha=$ const the same convergence properties in space and time are found as in flat geometries with periodic boundary conditions Chorin (1969). 

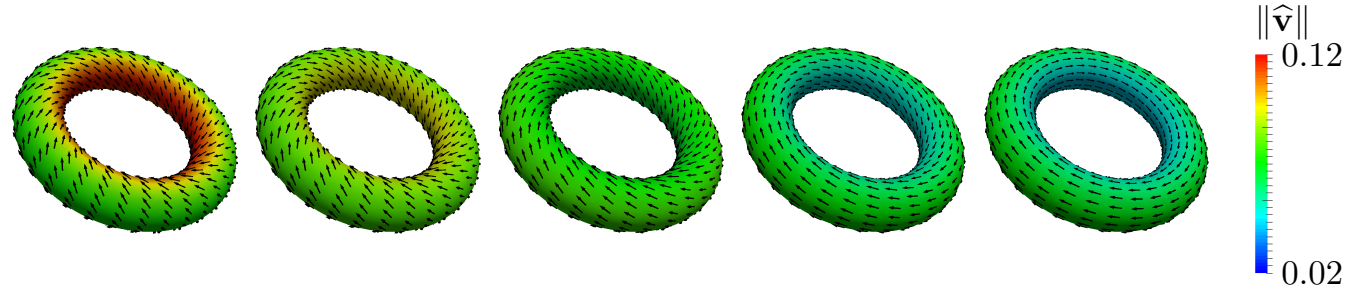

Figure 2: Numerical solution of $\widehat{\mathbf{v}}=-\boldsymbol{\nu} \times \widehat{\mathbf{w}}$ at $t=0,2,10,30$ and 60 (left to right). The color indicates the absolute value of the velocity $\widehat{\mathbf{v}}$. The arrows are rescaled for better visualization.
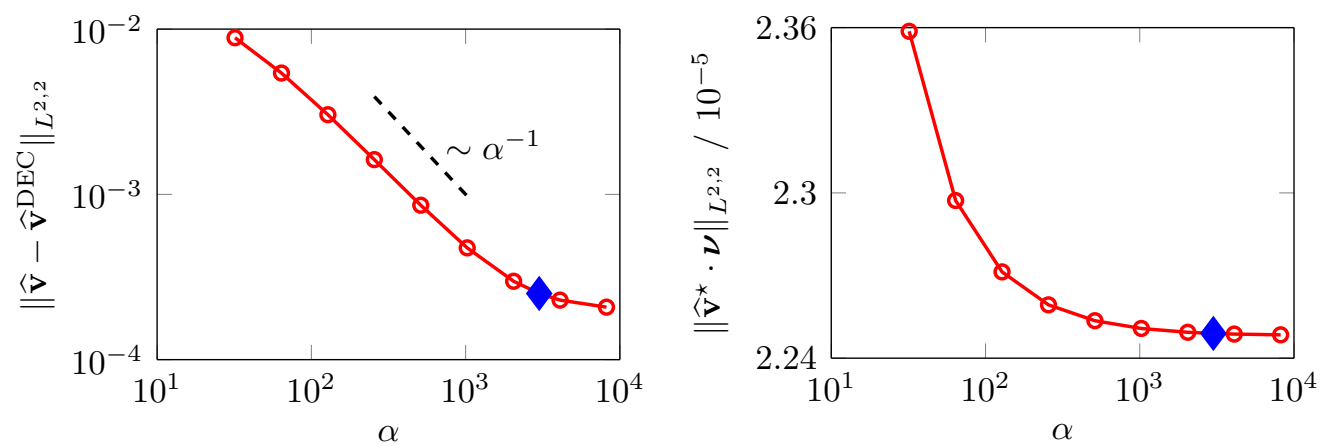

Figure 3: $L^{2,2}$ norm of the error between the present velocity field $\widehat{\mathbf{v}}$ and the velocity field $\widehat{\mathbf{v}}^{\mathrm{DEC}}$ computed with DEC against the penalty parameter $\alpha$ (left) and $L^{2,2}$ norm of the normal component of the rescaled velocity field $\widehat{\mathbf{v}}^{*}=\widehat{\mathbf{v}} /\|\widehat{\mathbf{v}}\|_{L^{2}}$ against the penalty parameter $\alpha$ (right). The first superscript index denotes the $L^{p}$ norm regarding time $t$ and the second superscript index denotes the spatial $L^{p}$ norm. The blue diamond indicates the penalty parameter $\alpha$ used for visualization in figure 2 and in the following examples. 


\section{Results}

The Poincaré-Hopf theorem relates the topology of the surface to analytic properties of a vector field on it. For vector fields $\mathbf{v} \in \mathrm{T} \mathcal{S}$ with only finitely many zeros (defects) it holds that $\sum_{\mathbf{x} \in \mathbf{v}^{-1}(\mathbf{0})} \operatorname{Ind}_{\mathbf{x}} \mathbf{v}=2-2 g(\mathcal{S})$ with $\operatorname{Ind}_{\mathbf{x}} \mathbf{v}$ the index or winding number of $\mathbf{x}$ for $\mathbf{v}$ and $g(\mathcal{S})$ the genus of the surface $\mathcal{S}$. To highlight this relation we consider $n$-tori for $n=1,2,3$ with genus 1,2 and 3 , respectively. Obviously, the simulation results have to fulfill the PoincaréHopf theorem in each time step, but they will also provide a realization of the theorem which depends on geometric properties and initial condition. Similar relations have already been considered for surfaces with $g(\mathcal{S})=0$ in Reuther and Voigt (2015); Nitschke et al. (2017).

A general form of a levelset function for a $n$-torus can be written as $L(\mathbf{x})=\prod_{i=1}^{n} T\left(\mathbf{x}-\mathbf{m}_{i}\right)-(n-1) \delta$ with a constant $\delta>0$ and the midpoints of the tori $\mathbf{m}_{i} \in \mathbb{R}^{3}$ for $i=1, \ldots, n$. In the following examples we consider the fully discrete scheme for Problem 2 and use $\operatorname{Re}=10, \tau=0.1, \alpha=3000$, $R=1$ and $r=0.5$. For the Gaussian curvature $\kappa$ we use the analytic formula. The initial condition is considered to be $\mathbf{v}_{0}=\operatorname{rot}_{\mathcal{S}} \psi_{0}=\boldsymbol{\nu} \times \operatorname{grad}_{\mathcal{S}} \psi_{0}$ with $\psi_{0}=\frac{1}{2}(x+y+z)$ which ensures the incompressibility constraint.

Figure 4 (top) shows the time evolution on the 1-torus with $\mathbf{m}_{1}=\mathbf{0}$. The initial state has four defects, two vortices with $\operatorname{Ind}_{\mathbf{x}} \mathbf{v}=+1$, indicated as red dots, and two saddles with $\operatorname{Ind}_{\mathbf{x}} \mathbf{v}=-1$, indicated as blue dots (one vortex and one saddle are not visible). These defects annihilate during the evolution. The final state is again a Killing vector field without any defects.

For $n>1$ the rotational symmetry is broken and Killing vector fields are no longer possible. We thus expect dissipation of the kinetic energy and convergence to $\mathbf{v}=\mathbf{0}$ for any initial condition. Figure 4 (middle) shows the time evolution on a 2-torus where we have used the midpoints $\mathbf{m}_{1}=$ $(-1.2,0,0)$ and $\mathbf{m}_{2}=-\mathbf{m}_{1}$ as well as $\delta=1$. The initial state has two vortices and four saddles and thus $\sum_{\mathbf{x} \in \mathbf{v}^{-1}(\mathbf{0})} \operatorname{Ind}_{\mathbf{x}} \mathbf{v}=-2$. Two vortex-saddle pairs annihilate each other and the final defect configuration consists of two saddles located at the center of the 2-torus (one is not visible). The velocity field decays towards $\mathbf{v}=\mathbf{0}$. Figure 4 (bottom) shows the time evolution on a 3 -torus with midpoints $\mathbf{m}_{1}=(-1.2,-0.75,0), \mathbf{m}_{2}=(1.2,-0.75,0)$ and $\mathbf{m}_{3}=(0,1.33,0)$ as well as $\delta=10$. Initially we have three vortices and seven saddles and thus $\sum_{\mathbf{x} \in \mathbf{v}^{-1}(\mathbf{0})} \operatorname{Ind}_{\mathbf{x}} \mathbf{v}=-4$, which is also fulfilled for the final defect configuration with two vortices and six saddles at the center of the 3-torus (one vortex and three saddles are not visible). Again the velocity 


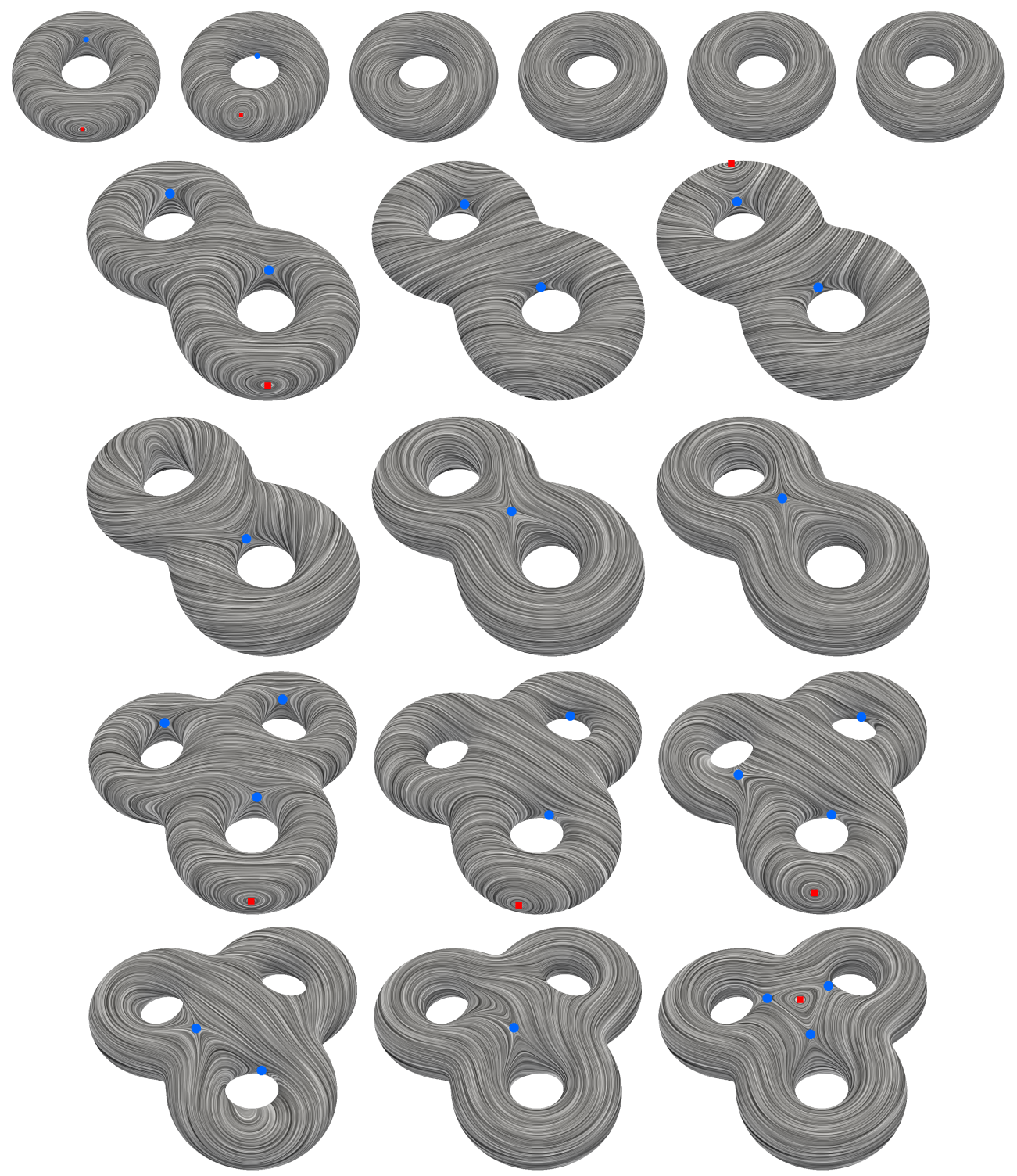

Figure 4: Numerical solution of $\widehat{\mathbf{v}}=-\boldsymbol{\nu} \times \widehat{\mathbf{w}}$ for the 1-torus (top row) at $t=0,5,10$, 15,25 and 100 (left to right), the 2-torus (2nd and 3rd row) at $t=0,10,20,30,50$ and 100 (left to right) as well as the 3-torus (4th and 5th row) at $t=0,10,20,30,50$ and 100 (left to right) visualized as noise concentration field aligned to the velocity field $\widehat{\mathbf{v}}$. The red squares and blue circles are indicating +1 defects (vortices) and -1 defects (saddles), respectively. The full evolution for the three examples is provided in the supplementary material. 

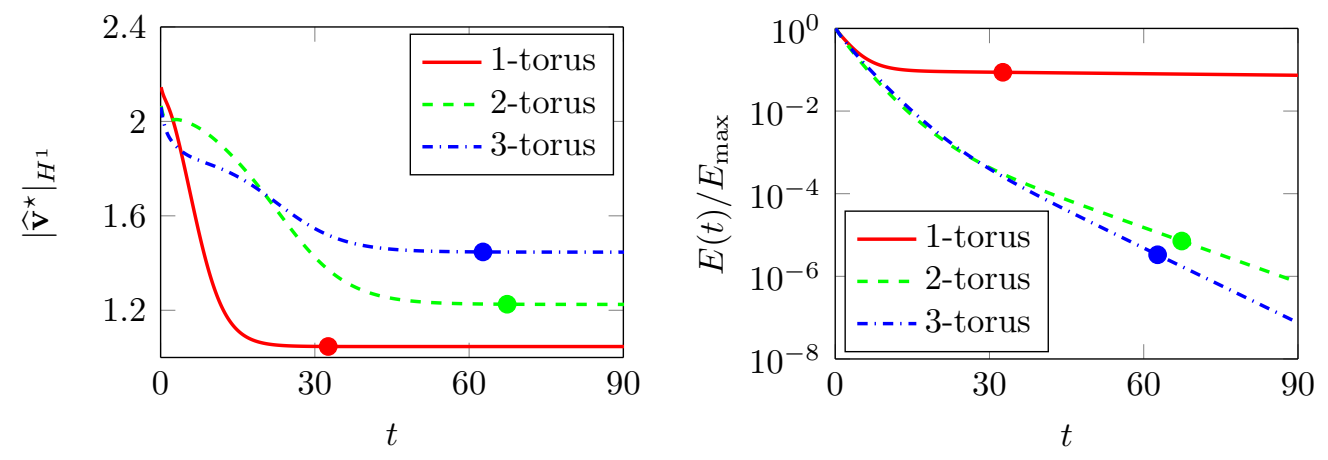

Figure 5: $H^{1}$ semi-norm of the rescaled velocity field $\widehat{\mathbf{v}}^{*}=\widehat{\mathbf{v}} /\|\widehat{\mathbf{v}}\|_{L^{2}}$ against time $t$ (left) and normalized kinetic energy $E / E_{\max }$ against time $t$ (right), where $E_{\max }$ is the maximum value of the kinetic energy $E$ over time. The colored dots are indicating the time points at which the defects reach their final position and only viscous dissipation takes place or a Killing vector field is formed. We identify these points if the decay rate of the $H^{1}$ semi-norm of the rescaled velocity field $\widehat{\mathbf{v}}^{*}$ reaches $0.001 \%$ of its maximum value over time.

field decays towards $\mathbf{v}=\mathbf{0}$.

To show the differences in the evolution on the $n$-tori before and after the final defect configuration is reached we consider the $H^{1}$ semi-norm of the rescaled velocity field $\widehat{\mathbf{v}}^{*}=\widehat{\mathbf{v}} /\|\widehat{\mathbf{v}}\|_{L^{2}}$. If the defects do not move this quantity is constant. Figure 5 shows the evolution over time together with the decay of the kinetic energy $E=\frac{1}{2} \int_{\mathcal{S}}\|\widehat{\mathbf{v}}\|^{2} d \mathcal{S}$.

These results clearly show the strong interplay between topology, geometric properties and defect positions.

\section{Conclusions}

We have proposed a discretization approach for the incompressible surface Navier-Stokes equation on surfaces with arbitrary genus $g(\mathcal{S})$. The approach only requires standard ingredients which most finite element implementations can offer. It is based on a reformulation of the equation in Cartesian coordinates of the embedding $\mathbb{R}^{3}$, penalization of the normal component, a Chorin projection method and discretization is space by globally continuous, piecewise linear Lagrange surface finite elements for each component. A further rotation of the velocity field leads to a drastic reduction of the complexity of the equation and the required computing time. The fully discrete scheme is described in detail and its accuracy validated against a DEC solution on a 1-torus, which was considered in Nitschke et al. (2017). The interesting 
interplay between the topology of the surface, its geometric properties and defects in the flow field are shown on $n$-tori for $n=1,2,3$.

Even if the formulation of the incompressible surface Navier-Stokes equation is relatively old Scriven (1960); Ebin and Marsden (1970); Mitrea and Taylor (2001), numerical treatments on general surfaces are very rare. We are only aware of the DEC approach in Nitschke et al. (2017) and therefore expect the proposed approach to initiate a broader use and advances in the mentioned applications in Section 1. We further expect it to be the basis for further developments, e.g. coupling of the surface flow with bulk flow in two-phase flow problems, as, e.g. considered in Reuther and Voigt (2016) using a vorticity-stream function approach or in Barrett et al. (2016) within an alternative formulation based on the bulk velocity and projection operators. Another extension considers evolving surfaces. With a prescribed normal velocity this has already been considered in Reuther and Voigt (2015), again using a vorticity-stream function approach. The corresponding equations are derived in Koba et al. (2017) using a global variational approach and in Miura (2017) as a thin-film limit. A mathematical derivation of the evolution equation for the normal component is still controversial. The derivation in Arroyo and DeSimone (2009) is based on local conservation of mass and linear momentum in tangential and normal direction, while the derivation in Jankuhn et al. (2017) is based on local conservation of mass and total linear momentum. The resulting equations differ. However, in the special case of a stationary surface, all these models coincide with the incompressible surface Navier-Stokes equation in eqs. (1) and (2).

Acknowledgements: This work is partially supported by the German Research Foundation through grant Vo899/11. We further acknowledge computing resources provided at JSC under grant HDR06 and at ZIH/TU Dresden.

\section{References}

Abraham, R., Marsden, J., Ratiu, T., 1988. Manifolds, Tensor Analysis, and Applications. No. 75 in Applied Mathematical Sciences. Springer.

Arroyo, M., DeSimone, A., 2009. Relaxation dynamics of fluid membranes. Physical Review E 79, 031915.

Barrett, J., Garcke, H., Nürnberg, R., 2015. Numerical computations of the dynamics of fluidic membranes and vesicles. Physical Review E 92, 052704. 
Barrett, J. W., Garcke, H., Nürnberg, R., 2016. A stable numerical method for the dynamics of fluidic membranes. Numerische Mathematik 134, 783822 .

Bertalmio, M., Cheng, L.-T., Osher, S., Sapiro, G., 2001. Variational problems and partial differential equations on implicit surfaces. Journal of Computational Physics 174, 759-780.

Bothe, D., Prüss, J., 2010. On the two-phase Navier-Stokes equations with Boussinesq-Scriven surface. Journal of Mathematical Fluid Mechanics 12, $133-150$.

Chorin, A., 1968. Numerical solution of the Navier-Stokes equations. Math. Comp. 22, 745-762.

Chorin, A., 1969. On the convergence of discrete approximations to the Navier-Stokes equations. Math. Comp. 23, 341-353.

Dörries, G., Foltin, G., 1996. Energy dissipation of fluid membranes. Physical Review E 53, 2547-2550.

Dziuk, G., Elliott, C. M., 2007a. Finite elements on evolving surfaces. IMA Journal of Numerical Analysis 27, 262-292.

Dziuk, G., Elliott, C. M., 2007b. Surface finite elements for parabolic equations. Journal of Computational Mathematics 25, 385-407.

Dziuk, G., Elliott, C. M., 2008. Eulerian finite element method for parabolic pdes on implicit surfaces. Interfaces and Free Boundaries 10 (1), 119-138.

Dziuk, G., Elliott, C. M., 2013. Finite element methods for surface PDEs. Acta Numerica 22, 289-396.

Ebin, D. G., Marsden, J., 1970. Groups of diffeomorphisms and the motion of an incompressible fluid. Annals of Mathematics 92, 102-163.

Elcott, S., Tong, Y., Kanso, E., Schröder, P., Desbrun, M., 2007. Stable, circulation-preserving, simplicial fluids. ACM Transactions on Graphics 26,4 . 
Greer, J. B., Bertozzi, A. L., Sapiro, G., 2006. Fourth order partial differential equations on general geometries. Journal of Computational Physics 216, 216-246.

Hansbo, P., Larson, M. G., Larsson, K., 2016. Analysis of Finite Element Methods for Vector Laplacians on Surfaces. arXiv:1610.06747.

Hu, D., Zhang, P., E, W., 2007. Continuum theory of a moving membrane. Physical Review E 75, 041605.

Jankuhn, T., Olshanskii, M. A., Reusken, A., 2017. Incompressible fluid problems on embedded surfaces: Modeling and variational formulations. arXiv:1702.02989.

Koba, H., Liu, C., Giga, Y., 2017. Energetic variational approaches for incompressible fluid systems on an evolving surface. Quart. Appl. Math. 75, 359-389.

Mitrea, M., Taylor, M., 2001. Navier-Stokes equations on Lipschitz domains in Riemannian manifolds. Mathematische Annalen 321, 955-987.

Miura, T.-H., 2017. On singular limit equations for incompressible fluids in moving thin domains. arXiv:1703.09698.

Mullen, P., Crane, K., Pavlov, D., Tong, Y., Desbrun, M., 2009. Energypreserving integrators for fluid animation. ACM Transactions on Graphics 28,38 .

Nestler, M., Nitschke, I., Praetorius, S., Voigt, A., 2017. Orientational order on surfaces: The coupling of topology, geometry, and dynamics. Journal of Nonlinear Science, DOI:10.1007/s00332-017-9405-2.

Nitschke, I., Reuther, S., Voigt, A., 2017. Discrete exterior calculus ((EC) for the surface Navier-Stokes equation. In: Bothe, D., Reusken, A. (Eds.), Transport Processes at Fluidic Interfaces. Springer, pp. 177-197.

Nitschke, I., Voigt, A., Wensch, J., 2012. A finite element approach to incompressible two-phase flow on manifolds. Journal of Fluid Mechanics 708, 418-438. 
Padberg-Gehle, K., Reuther, S., Praetorius, S., Voigt, A., 2017. Transfer operator-based extraction of coherent features on surfaces. In: Carr, H., Garth, C., Weinkauf, T. (Eds.), Topological Methods in Data Analysis and Visualization IV: Theory, Algorithms, and Applications. Springer, pp. 283-297.

Rätz, A., Voigt, A., 2006. PDE's on surfaces: A diffuse interface approach. Communications in Mathematical Sciences 4, 575-590.

Reuther, S., Voigt, A., 2015. The interplay of curvature and vortices in flow on curved surfaces. Multiscale Modeling \& Simulation 13, 632-643.

Reuther, S., Voigt, A., 2016. Incompressible two-phase flows with an inextensible Newtonian fluid interface. Journal of Computational Physics 322, 850-858.

Sasaki, E., Takehiro, S., Yamada, M., 2015. Bifurcation structure of twodimensional viscous zonal flows on a rotating sphere. Journal of Fluid Mechanics 774, 224-244.

Scriven, L. E., 1960. Dynamics of a fluid interface equation of motion for Newtonian surface fluids. Chemical Engineering Science 12, 98-108.

Secomb, T. W., Skalak, R., 1982. Surface flow of viscoelastic membranes in viscous fluids. The Quarterly Journal of Mechanics and Applied Mathematics 35, 233-247.

Stöcker, C., Voigt, A., 2008. Geodesic evolution laws - a level-set approach. SIAM Journal on Imaging Sciences 1, 379-399.

Vaxman, A., Campen, M., Diamanti, O., Panozzo, D., Bommes, D., Hildebrandt, K., Ben-Chen, M., 2016. Directional field synthesis, design and processing. In: EUROGRAPHICS - STAR. Vol. 35. pp. 1-28.

Vey, S., Voigt, A., 2007. AMDiS: Adaptive multidimensional simulations. Computing and Visualization in Science 10, 57-67.

Witkowski, T., Ling, S., Praetorius, S., Voigt, A., 2015. Software concepts and numerical algorithms for a scalable adaptive parallel finite element method. Advances in Computational Mathematics 41, 1145-1177. 